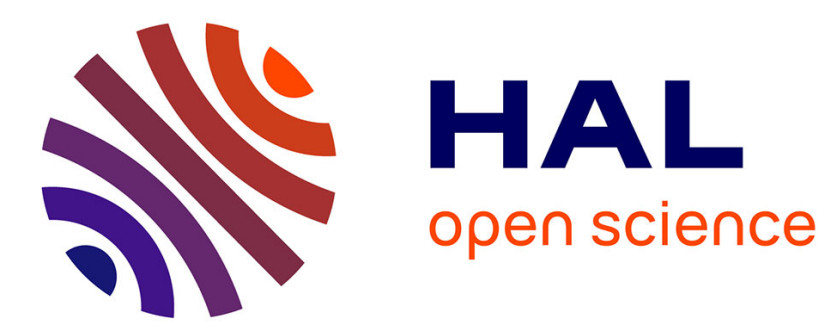

\title{
Use and misuse of discrete character data for morphospace and disparity analyses
}

Sylvain Gerber

\section{To cite this version:}

Sylvain Gerber. Use and misuse of discrete character data for morphospace and disparity analyses.

Palaeontology, 2019, 62 (2), pp.305-319. 10.1111/pala.12407 . hal-02340296

\section{HAL Id: hal-02340296 \\ https://hal.sorbonne-universite.fr/hal-02340296}

Submitted on 30 Oct 2019

HAL is a multi-disciplinary open access archive for the deposit and dissemination of scientific research documents, whether they are published or not. The documents may come from teaching and research institutions in France or abroad, or from public or private research centers.
L'archive ouverte pluridisciplinaire HAL, est destinée au dépôt et à la diffusion de documents scientifiques de niveau recherche, publiés ou non, émanant des établissements d'enseignement et de recherche français ou étrangers, des laboratoires publics ou privés. 


\section{USE AND MISUSE OF DISCRETE CHARACTER DATA FOR MORPHOSPACE AND}

DISPARITY ANALYSES

Sylvain Gerber

Institut Systématique Evolution Biodiversité (ISYEB), Muséum national d'Histoire naturelle, CNRS, Sorbonne

Université, EPHE, 45 rue Buffon, CP 50, 75005 Paris, France; sylvain.gerber@mnhn.fr

Running head: Discrete character data in disparity analyses

Abstract

The measurement of morphological variation in macroevolutionary studies is increasingly based on morphospaces constructed from discrete character data. This trend mostly results from the appropriation of phylogenetic data matrices as character spaces for carrying out disparity analyses. Phylogenetic matrices provide morphological descriptions of taxa as combinations of character states and thus appear - if not conceptually, at least mathematically - comparable to discrete character datasets found in numerical taxonomy or built for disparity purposes. Hence, phylogenetic matrices seem to constitute an abundant source of data readily available for morphospace analyses. Discrete character spaces have been generally described as more flexible than morphospaces capturing continuous shape variation. The discrete coding of morphology allows morphospaces to accommodate more disparate morphologies and the

24 ability of discrete character schemes to handle missing data is also often emphasized. This

25 flexibility comes at a cost, however. Multivariate ordinations of such spaces often provide 
deceptive visualizations and may invite the use of inappropriate methodologies for their exploration. The high amount of missing data that typifies many phylogenetic datasets is also problematic for the measurement of dissimilarity among taxa and can therefore be detrimental to the assessment of morphological disparity. In the present contribution, the properties of discrete character spaces are described and common pitfalls discussed. Graphical and methodological approaches are suggested to circumvent or limit their impact, and greater caution is recommended when using discrete character data for morphospace and disparity inferences.

Key words: character space, phylogenetic data matrices, disparity, macroevolution, missing data, morphological hypercube. 
The morphological disparity of a clade can be defined as a measure of the degree of morphological distinctness exhibited by its constitutive taxa (for reviews, see Foote 1997; Wills 2001; Erwin 2007; Wagner 2010; Hopkins and Gerber 2017). Its study has been pioneered by palaeobiologists in the 90's (e.g. Gould 1991; Foote 1993), but the concept has since reached prominence as a macroevolutionary measure of morphological variation in both palaeontological and neontological contexts (e.g. Neige 2003; Ricklefs 2004; Chartier et al. 2017). Methodologically, morphological disparity is estimated from the quantitative description of the distribution of taxa in morphospace, the space spanned by the set of morphological variables used to describe and compare the taxa studied.

Diverse kinds of morphospaces can be built depending on the descriptors used to characterise the sample of morphologies considered. In what follows, I focus on discrete character spaces. These morphospaces have recently risen in popularity in palaeobiology, particularly among vertebrate specialists, as a consequence of the idea of co-opting phylogenetic data matrices as a source of morphological data for disparity analysis (e.g. Brusatte et al. 2008, 2011; Prentice et al. 2011; Thorne et al. 2011; Butler et al. 2012; Ruta et al. 2013; Hetterington et al. 2015; Romano 2017). Lloyd (2016) provided a recent account of the strengths and challenges associated with the use of discrete character data in studies of disparity and rates of morphological evolution. The theoretical and empirical features of discrete character spaces do not seem to have been much discussed however, and how these features affect morphospace and disparity studies has therefore been largely overlooked. In the present contribution, I describe and illustrate some properties of discrete character spaces and highlight potential pitfalls with the use of such spaces in disparity studies within current analytical frameworks. Although some of these issues do not seem to have ideal solutions for now, some suggestions are made to lower their impact on the interpretation of morphospace patterns and on estimates of morphological disparity. 
The discussion mostly revolves around two points in need of further attention: the use of reduced-space ordination methods to visualize discrete character spaces and the large amount of missing data that typifies many phylogenetic datasets. Regarding the first point, it is often tempting to try to visualize the morphospaces we are working with. The use of ordination methods on discrete character spaces however leads to visualizations that can be prone to misinterpretations. It also tends to cause confusions between the morphospace itself and its ordination, inviting the use of inappropriate methods for analysing morphospace patterns. A hypercube graph example is developed to illustrate these aspects. The second point on missing data reflects a pressing concern given the widespread use of phylogenetic datasets for disparity analyses. Abundant missing entries have a substantial and detrimental effect on the assessment of morphological dissimilarity among specimens and can therefore strongly bias disparity estimates, in particular if those are calculated from ordinations of deficient pairwise distance matrices. Graphical options and weighting approaches are suggested to account for the uneven quality of specimen scoring.

\section{DISCRETE CHARACTER DATA AND PHYLOGENETIC DATA MATRICES}

Discrete character data have long been used as a means of quantifying morphological variation, including in the early years of the disparity research program (e.g. Foote 1992; Thomas and Reif 1993). Discrete coding corresponds to the morphological description of each specimen in a sample as a sequence of character states. The characters can possess two or more states, corresponding to binary and multistate characters respectively. In the latter case, the different states can be treated as ordered or unordered depending on the underlying mode of variation that is assumed. 
101 One of the main advantages of discrete character schemes compared to other descriptors lies

102 in their ability to accommodate a broader range of morphologies within a single analysis.

103 Indeed, fairly disparate morphological structures, which can be challenging for morphometric

104 approaches, can usually be more easily analysed within a discrete character framework.

105 Discrete coding also offers the possibility of including morphological features that are only

106 present in a subset of the sample of specimens (a context that also precludes many alternative

107 morphometric methods). The study of evolutionary novelties is an important research

108 program in macroevolution (e.g. Wagner and Lynch 2010; Erwin 2017) and the ability to

109 account for the origination of novel morphological features (e.g. via a presence/absence

110 coding scheme) is thus central to disparity. Discrete character data therefore appear especially

111 relevant for studies at high taxonomic ranks where morphological variation is expected to be

112 substantial and homology difficult to define uniformly across the taxa sampled. Discrete data

113 are also useful if the morphological phenotypes considered include articulated or

114 disconnected structures, such as the different bones of a skeleton. This flexibility generally

115 comes at the expense of relatively cruder descriptions of morphologies, but careful coding

116 schemes can still allow informative accounts of the morphological traits deemed relevant for a

117 study.

118 Phylogenetic data matrices are instances of discrete character data and as such they have been

119 increasingly considered as the basis for morphospace and disparity analyses in recent years.

120 One could nevertheless expect them to differ from the discrete character datasets that are

121 specifically assembled for the purpose of disparity analyses. Phylogenetic data matrices are

122 built for phylogenetic purposes and this has logical implications for the definition and coding

123 of characters (e.g. Estabrook et al. 1975), notably regarding homoplasy (convergence and

124 reversal) and autapomorphies (derived character state restricted to a unique terminal taxon).

125 The aim of phylogenetics is to establish branching orders and group taxa into evolutionarily 
coherent clades. Therefore, morphological features that would be known or anticipated to be

127 highly homoplastic would not in principle be considered as suitable characters and included in

128 a phylogenetic dataset. An opposite view on homoplasy is generally held in the field of

129 disparity (Gould 1991; Foote 1996). Far from being nuisances, convergences and reversals

130 are seen as important evolutionary phenomena in studies of morphological disparity (see

131 Foote 1996 for a discussion on total versus net changes) and their consideration, in

132 conjunction with a phylogenetic hypothesis, improve the interpretations of evolutionary

133 patterns. Autapomorphic characters are uninformative in parsimony analysis, which is still the

134 most widespread approach in morphology-based phylogenetic inference (autapomorphies are

135 nonetheless informative in maximum likelihood and Bayesian approaches; Lewis 2001). One

136 should therefore expect them to be rarely found in phylogenetic datasets. The view of their

137 role in disparity studies ranges from central importance (Gould 1991) to negligible (Cisneros

138 and Ruta 2010; Ruta and Wills 2016) but for distinct conceptual and methodological reasons

139 (see below).

140 Hence, phylogenetic and disparity datasets, respectively aiming at grouping and

141 distinguishing taxa, in principle reflect two different modes of character matrix construction.

142 In a hypothetical phylogenetic dataset with no homoplasy and no autapomorphies, two

143 terminal sister taxa (OTUs) share the same sequence of character states. They are, as scored,

144 morphologically undistinguishable, which is not pertinent from a disparity viewpoint. The use

145 of phylogenetic datasets for disparity purposes thus implicitly relies on the expectation of

146 some erroneous assessments of character state homology and/or on the presence of

147 parsimony-uninformative characters.

148 Empirically, it appears that discrete character datasets built for phylogenetic and disparity

149 purposes do not in fact greatly differ from each other regarding the nature of the characters

150 that they include. Based on measures of HER (Homoplasy Excess Ratio), Hughes et al. 
151 (2013) showed that phylogenetic and disparity datasets on average exhibit comparable

152 degrees of homoplasy, even though the small sample of datasets investigated did not allow

153 further comparison of the statistical distributions of HER for the two kinds of datasets.

154 Studies of rates of character change in various groups have also shown that rate distributions

155 are better fitted by gamma and lognormal models rather than by uniform (single-rate) models

156 (Wagner et al. 2006; Wagner 2012; Harrison and Larsson 2015). These non-uniform

157 distributions indicate significant rate heterogeneities among characters and suggest that

158 phylogenetic data matrices commonly include homoplastic characters. Phylogenetic and

159 disparity datasets also appear comparable with respect to the presence of autapomorphic

160 characters. Most phylogenetic data matrices include autapomorphic characters indicating that

161 phylogeneticists do not deliberately exclude them regardless of their relevance to the method

162 of phylogenetic inference subsequently used (P. Wagner, pers. comm., based on a meta-

163 analysis of 188 matrices of invertebrate clades showing that most matrices have at least one

164 autapomorphic binary character, and nearly all have at least one autapomorphic character

165 state on either a binary or multistate character).

166 Whether the two distinct research questions underlying the construction of phylogenetic and

167 disparity datasets lead to comparable matrices or not, the use of the former as a surrogate for

168 the latter still implies a series of reformatting steps. A phylogenetic data matrix cannot be

169 directly co-opted without at least a few changes (see Hughes et al. 2013). For instance, the

170 row corresponding to the outgroup needs to be removed in most cases. The question that

171 motivated the construction of the phylogenetic data matrix might also have had an influence

172 on the taxonomic coverage and (sub)sampling effort carried out, and it is important to assess

173 the representativeness of the sampled OTUs for a disparity analysis meant to characterize the

174 clade they belong to. Besides, it is also not uncommon for OTUs in a phylogenetic data

175 matrix to represent different taxonomic ranks and some matrix rows might need to be 
amalgamated to level these differences and homogenise the dataset. Finally, phylogenetic data

177 matrices conducive to phylogenetic trees with low level of homoplasy might indicate a stringent selection of characters and the possibility of expanding the character set in the prospect of a disparity analysis.

Hence, a phylogenetic data matrix can form a basis for the elaboration of a discrete character

181 space, but the taxa and characters enclosed generally need critical re-evaluation. The characters eventually gathered generate a set of possibilities for morphological variation ("possibilities" in a combinatorial but not necessarily biological sense). This set can be finite or infinite depending on the definition of characters and their implied number of states (e.g., Hoyal Cuthill 2015). The next step is to define relationships among these possible morphological options, traditionally based on measures of morphological (dis)similarity, in order to generate a morphological space.

\section{MEASURING DISSIMILARITY AND THE MORPHOLOGICAL HYPERCUBE}

Hereafter, only binary characters are considered. This allows a simpler derivation of the observations and conclusions that are equally valid for geometrically more complex morphospaces built from datasets that include multistate characters. For convenience, it is also assumed that there is no missing data (this issue is dealt with in a following section) and that all characters can be treated equally. This last point means that all characters are given

197 the same weight when estimating pairwise dissimilarity. Note that this equal weighting of 198 characters is unlikely to be biologically meaningful given the anatomical diversity of characters typically combined, but this is the approach usually taken in empirical studies (see 
With discrete character data, the measurement of dissimilarity between two taxa is based on

202 the number of matches and mismatches between their scored character states. For binary

203 characters, this is equivalent to measuring the Hamming distance between the two sequences

204 of character states. For instance, for a set of $p=6$ binary characters, the OTUs A $(1,0,0,1,0$,

205 1) and B (1, 1, 0, 0, 0, 1) have 4 matches (characters 1, 3, 5, and 6) and 2 mismatches

206 (characters 2 and 4). The Hamming distance between A and B is thus 2. The distance between

207 any possible pairs of morphologically distinct OTUs ranges from 1 to $p$. The Hamming

208 distance satisfies the properties of equality, positivity, symmetry, and triangle inequality, and

209 it is therefore a metric on the set of all sequences of binary character states of length $p$. The

210 resulting discrete character space is a Hamming cube. If one considers the $p$ states of all

211 possible sequences as coordinates in $\mathbb{R}^{p}$, one obtains a Euclidean representation of the discrete

212 character space as a $p$-dimensional unit hypercube. A hypercube is the generalization of the

213 concept of cube to higher dimensions. Each sequence occupies one of the vertices of the

214 hypercube, and the distance between two sequences is the sum of unit-length edges separating

215 their vertices (as opposed to the Euclidean distance between vertices). In this context, the

216 morphospace is the spatial representation of taxa relative to an underlying set of possibilities

217 for morphological variation. With $p$ binary characters, this set of possibilities is the $2^{p}$

218 combinations of the character states corresponding to the $2^{p}$ vertices of the hypercube.

219 Some geometric properties of such morphospaces can be easily inferred. For instance, adding

220 an autapomorphic character to a dataset adds one dimension to the morphological hypercube,

221 but it doubles the set of options for morphological variation: $p+1$ binary characters give $2^{(p+1)}$

222 sequences, that is, $2 \times 2^{p}$ possible combinations of characters. The $(p+1)$-hypercube has now

223 twice the number of vertices, but only one of these extra $2^{p}$ vertices is (and can be) occupied.

224 This is not necessarily problematic but it must be kept in mind if one uses quantitative

225 approaches whose performances are sensitive to morphospace dimensionality. Geometrically, 
an autapomorphy adds a dimension that separates the taxon that possesses it from all the other taxa of the clade. If autapomorphies are uniformly distributed across OTUs, their overall effect is to increase pairwise distances by pushing OTUs away from each other in orthogonal directions (in the Euclidean representation of the hypercube). In this case, their interest is limited for autapomorphies will not qualitatively affect the morphospace structure and relative 231 measures of disparity extracted from it (Cisneros and Ruta 2010). A more restricted and 232 informative distribution of autapomorphies might also have a limited effect on overall disparity patterns inasmuch as their individual contributions to dissimilarity measures is relative to the total number of characters, but their interest rests upon their ability to single-out remarkable taxa and document the structure of morphospace occupation (Gould 1991; Foote 1997).

Many other geometric features of discrete character spaces are not as easily grasped and highdimensional objects can be challenging to our intuition (e.g. Abbott 1884). Visualisation techniques such as projections onto lower-dimensional spaces are therefore frequently employed and it is important to assess the effect of such approaches on our understanding and 241 interpretation of morphospace patterns.

\section{MORPHOSPACE ORDINATION AND VISUALIZATION}

246 Morphospace visualization is often considered as an important step for illustrating and 247 discussing morphological patterns. Like most morphospaces however, discrete character 248 spaces have a high number of dimensions and two- or three-dimensional representations of 249 morphospaces consequently offer only a partial view of the amount and structure of morphospace occupation. Great care is therefore required when describing morphospace 
251 patterns from a reduced number of axes. This is especially true for discrete character spaces

252 for which usually only a very small fraction of the variation is captured by the first axes of the 253 multivariate ordination.

254 For discrete character data, it is customary to visualize a morphospace by carrying out a 255 Principal Coordinate Analysis (PCoA) on the pairwise dissimilarity matrix $\mathbf{D}$ (an alternative 256 not discussed here is Non-Metric Multidimensional Scaling, but the method aims at 257 preserving ordering relations rather than distances among the objects it ordinates). PCoA provides a Euclidean representation of a dissimilarity matrix (Gower 1966), here analogous to the hypercube geometry described above. The principal coordinates generated (at most $n-1$ where $n$ is the number of OTUs) are functions of the original variables (the morphological 261 characters), as conveyed by the choice of the distance measure. If $\mathbf{D}$ is not Euclidean, because 262 of the distance measure chosen (or because of missing data), negative eigenvalues will be 263 produced. A transformation of $\mathbf{D}$ is therefore recommended prior to its ordination (e.g. square root transformation).

265 The resulting depiction of the morphospace is the set of taxa displayed as points in the space 266 of the ordination, a generic representation common to all types of morphological descriptors. 267 Importantly however, this ordination is an embedding of the true discrete character space 268 (morphological hypercube or similar but more complex discrete spaces) in a continuous 269 Cartesian coordinate system, that of the principal coordinate axes. Unfortunately, this discrete 270 structure is difficult to represent and is therefore often overlooked. Yet, conflating the 271 geometric properties of the morphospace with those of its ordination has non-trivial 272 consequences for the interpretation of morphological patterns and for the choice of methods 273 employed to explore morphospaces.

274 A small example is presented in Figure 1 to illustrate these points. It is based on a subset of 275 the archosaur dataset from Brusatte et al. (2008). For the sake of the example, a stringent 
selection of characters had to be carried out in order to obtain a dataset where the OTUs

277 retained are scored using only binary characters and without missing data. The culling of rows

278 and columns from the original phylogenetic data matrix undoubtedly leads to an

279 uninformative dataset, but it remains relevant for the present illustrative purpose. The subset

280 considered includes 18 genera of crurotarsans coded for 10 binary characters (characters $\{2$,

$2813,4,9,19,21,22,24,25,45\}$ in Brusatte et al. 2008). As described above, dissimilarity

282 among genera is calculated as the Hamming distance and these pairwise distances are stored

283 in the distance matrix D. In the interest of visualization, a multivariate ordination of the

284 morphospace is produced by carrying out a PCoA of the square root of $\mathbf{D}$. Figure 1A shows

285 the traditional representation of the morphospace with taxa abstracted as points in the

286 principal coordinate space.

287 Two related questions are generally investigated or discussed when considering morphospace

288 patterns. One concerns the overall amount of morphospace being occupied by a clade and the

289 reasons for the existence of unoccupied regions. Discussions of this problem arose from

290 Raup's seminal work on the unequal filling of the shell coiling morphospace (Raup 1966) and

291 are central to the field of theoretical morphology (e.g. McGhee 2006). The causes generally

292 invoked include the functional and developmental limits imposed on the variational properties

293 of phenotypes, ecological restrictions, and historical contingency (e.g. Raup 1972, Maynard

294 Smith et al. 1985; Erwin 2007; Oyston et al. 2015). The second research question aims at

295 dissecting the temporal and/or spatial dynamics of morphospace occupation and forms the

296 core of disparity analyses.

297 If the discrete character space is mistaken for its ordination, both these research questions can

298 be misdirected. A priori unoccupied regions visible on the principal coordinate ordination

299 might correspond to positions that do not belong to the discrete character space and therefore

300 do not call for any biological explanation. Only a finite and non-randomly located (and in fact 
quite structured) positions correspond to the hypercube vertices, i.e. to meaningful positions

302 in the actual morphospace. This is illustrated in Figure 1B, where the two-dimensional 303 projection of the entire architecture of the 10-hypercube is displayed, that is, its 1024 vertices 304 and its 5120 unit-length edges. To obtain this representation, all 1024 combinations of 305 character states needs to be enumerated, along with a record of their 10 neighbouring 306 combinations (those differing by only one character state change). With $p$ characters, there are $3072^{p}$ vertices and $2^{p-1} p$ edges though, and it becomes rapidly difficult to enumerate all possible options and represent the hypercube graphically. Figure 1C shows a simplified representation where only a small subset of vertices and edges are computed and displayed, defined in a way that ensures a complete connectedness of the observed OTUs. It emphasizes the strong visual 311 effect of the two-dimensional projection of a high-dimensional discrete morphospace. All the 312 segments depicted represent edges and have therefore the same unit length despite their 313 drastically variable projected lengths. Also, provided the distance matrix subjected to PCoA is

314 Euclidean, edges are either parallel (if they correspond to the same character) or orthogonal, 315 even if the ordination suggests otherwise. Spatial relationships among vertices inferred from 316 the ordination should therefore be treated with caution since they will be misleading in most 317 cases. This bias typically impacts graphical techniques such as convex hulls. The relative 318 surface areas of these polygons can be poor indicators of the relative disparity of the groups 319 they delineate in the ordination.

320 A possible way to visually circumscribe the morphospace within the space of principal 321 coordinates could be to consider the hypersphere it is inscribed in (as conveyed by Fig. 1B).

322 By definition, all vertices are at a distance $(\sqrt{ } p) / 2$ from the centroid of the hypercube. Hence, 323 one could draw the circle of radius $(\sqrt{ } p) / 2$ centred on the hypercube centroid to have an idea 324 of the size of the morphospace. The centroid position can be calculated as the average 325 principal coordinate scores of one of the observed vertices and of its diagonally opposite 
vertex (i.e. its opposite combination of character states). In practice however, the probability

327 of having a good correlation between the true and the projected maximum distances is low

328 (and decreases as $p$ increases) and the circle drawn will generally largely exceeds the two-

329 dimensional distribution of the vertices. An alternative is proposed below based on the pattern

330 of distribution of vertices.

331 Figure 1D illustrates the variation of the density of vertices under random orientations of the

332 hypercube along the first principal coordinate axis (here the hypercube is centred at the origin

333 of the principal coordinate system for convenience). It shows a density that decreases as one

334 moves away from the hypercube centroid (this can also be noticed in Fig. 1B). This pattern

335 has important implications: it means that even a uniform filling of the discrete morphospace

336 will lead to a non-uniform distribution of taxa in the two- or three-dimensional visualization

337 supplied by the PCoA (an orthogonal projection of the hypercube). Hence, density contour

338 lines, heatmap representations, and similar approaches found in spatial point pattern analysis

339 which might seem appropriate to document morphospace occupation (e.g. Ruta and Wills

340 2016) should be avoided or used with caution. More generally any method that relies on the

341 continuous coordinate system of the ordination might be prone to similarly misleading

342 description of morphological patterns.

343 The density of vertices along any principal coordinate axis is approximately distributed as

$344 N(0,0.25)$ (PCoA considers each binary character as a dimension of the Euclidean space, and

345 their individual variance for the entire population of vertices is 0.25 ). Frequency ellipses can

346 be used to delineate the bivariate distribution of the vertices in the plane of pairs of principal

347 coordinate axes. In the bivariate case, a circular ellipse of radius $\approx 1.224$ centred on the

348 hypercube centroid will on average enclosed 95 percent of the morphospace vertices (with

349 two degrees of freedom, $\chi_{2}^{2}(0.95) \approx 5.991$, so the radius of the circular ellipse is indeed

$350 \sqrt{(}(5.991 \times 0.25)$; see Fig. 1C). 
This sub-circular shape of the morphospace also explains the very low percentage of variance that is usually summarized by the first few principal coordinate axes. Hypercubes and related spaces do not show preferential directions of elongation (hence the smooth decrease of eigenvalues). Nevertheless, some datasets that are more structured and depart from a uniform filling of the hypercube can show a more efficient redistribution of variance following PCoA. Such a structured morphospace occupation can be due to strong phylogenetic signal, clustered sets of character state combinations (morphologically distinct groups of taxa), or, more problematically, non-random distribution of missing data.

\section{PHYLOMORPHOSPACE}

phylogenetic datasets naturally encourage the use of their phylogenetic content in the course of disparity analyses, and the usefulness of contrasting patristic and phenetic dissimilarities in disparity studies has been emphasized (e.g. Foote 1996; Wagner 1997). Here I restrict the discussion to the use of phylogenetic information to project the inferred tree onto the morphospace. This construction has been referred to as "phylomorphospace" (Sidlauskas 2008, but see Strelin et al. 2018 for a warning regarding the use of this term). It has been used to infer the placement of "ancestors" in the morphospace, assess the strength of the phylogenetic signal contained in a morphological dataset, and test for patterns of convergence (e.g. Klingenberg and Gidaszewski 2010; Stayton 2015).

Producing a phylomorphospace visualization implies determining the location of the internal nodes of the tree in the morphospace, i.e., estimating their phenotypic trait values, and therefore requires a model of trait evolution. Importantly, the meaningfulness of these estimates depends on the adequacy of the model chosen. Different methods are employed for 
inferring ancestral trait values depending on whether the phenotypic traits considered show

377 discrete or continuous modes of variation. In the case of discrete character spaces, it is therefore again critical to properly distinguish the discrete architecture of the morphospace from its ordination in a space equipped with a continuous coordinate system. Only methods devised for discrete traits (e.g. maximum parsimony or posterior probabilities calculated from 381 the likelihoods of ancestral states in Mk models; Cunningham et al. 1998; Hunt and Slater 382 2016) are appropriate for estimating ancestral character states in this context. Use of methods 383 for continuous traits (e.g., squared-change parsimony, maximum likelihood models based on Brownian motion) applied to the principal coordinate scores of OTUs will lead to the incorrect placement of ancestral nodes in the ordination (e.g. Ruta et al. 2013; Hopkins and

386 Smith 2015). Their positions will be undefined locations in the discrete morphospace and 387 subsequent analyses and interpretations relying on them will be flawed.

388 Figure 2 provides a simple theoretical illustration of this problem. Five OTUs (labelled A to

389 E) are coded with 8 binary characters. These include 3 parsimony informative characters that 390 support the phylogenetic reconstruction (Fig. 2A) and 5 autapomorphic characters that 391 distinguish the OTUs from each other. Character states at internal nodes are estimated using 392 maximum parsimony. As mentioned earlier, without autapomorphies and in the absence of 393 homoplasy, sister OTUs would be merged with their supporting node. For instance, OTUs A 394 and $\mathrm{B}$ and node $\mathrm{i}$ would all be described by the sequence $(1,0,0)$.

395 Figure 2B shows the morphological hypercube (8 dimensions) embedded in the Euclidean 396 space. The tips (OTUs) and the reconstructed internal nodes of the tree are located at their 397 respective vertices of the hypercube. The branches of the tree correspond to edges (or 398 sequences of edges in the general case) and are highlighted. This is a phylomorphospace 399 representation that accounts for the discrete nature of the characters. Figure $2 \mathrm{C}$ shows the 400 same hypercube and the same OTUs, but the distribution of internal nodes has been estimated 
using squared-change parsimony from the principal coordinate scores of the OTUs. The resulting depiction of the tree is substantially different from that of Figure $2 \mathrm{~B}$ and none of the inferred internal nodes belong to vertices of the hypercube. This representation is invalid because it fails to acknowledge the true, underlying morphospace.

Phylomorphospaces built from continuous traits usually provide easily interpretable bivariate 406 visualizations, with all internal nodes located within the convex hull defined by the OTUs and 407 a degree of branch-crossing indicative of the amount of homoplasy. Conversely, with discrete 408 characters, phylomorphospace visualizations tend to show very complex patterns from which 409 little can be inferred (e.g. Halliday and Goswami 2015). Figure 2D illustrates the geometry of 410 the phylomorphospace representation in the simple case considered. The parsimony 411 informative characters supporting the tree define a subspace within the discrete morphospace. 412 It is also a hypercube but of lower dimensionality (here it is a cube). As described earlier and 413 shown here, the autapomorphies push OTUs away from the subspace of parsimony 414 informative characters in orthogonal directions.

415 Brusatte et al. (2011) suggested the use of inferred ancestral nodes to derive phylogenetically 416 corrected disparity curves. They described that the addition of reconstructed ancestors leads to 417 an inflation of the morphospace, since these ancestors appear to fall outside the limits drawn 418 by the observed taxa, as outlined by means of convex hulls. This is in fact a misinterpretation 419 of the effect of the bivariate projection of the discrete morphospace and a drawback of the use 420 of convex hulls in a discrete character context. These ancestors do not represent any aberrant 421 or inexplicable combinations of character states but correspond to existing vertices on the 422 discrete morphospace. Their apparent peripheral distribution results from the geometry of the 423 morphospace (see Fig. 2B), the presence of homoplasy, and the distribution of the total set of taxa (observed and reconstructed) from which the ordination plane is defined. 


\section{THE PROBLEM OF MISSING DATA}

Unscored entries in discrete character data matrices is problematic both for phylogenetic inference (e.g. Platnick et al. 1991; Maddison 1993; Wilkinson 1995; but see Kearney 2002) and for disparity analyses. They correspond to either missing or inapplicable data, and are traditionally reported as '?' and '-', respectively. Missing and inapplicable character states are handled similarly by phylogenetic software such as PAUP* (Swofford 2003) or TNT (Goloboff et al. 2008), and within standard disparity pipelines. The distinction between the two is not negligible from a morphospace perspective however.

Inapplicable character states

Within current methodological frameworks, it does not seem advisable to retain in a disparity-

441 aimed dataset characters that happen to be inapplicable for one or more taxa in a sample.

442 These taxa do not have definable positions along the dimensions defined by the inapplicable 443 characters but instead occupy the complementary subspace of the morphospace. A distance 444 matrix can still be computed (albeit a deficient one) and ordinated by means of PCoA, but this 445 will artificially enforce the placement of these taxa in a space they do not technically reside 446 in. As it is sometimes done, disparity can even be calculated from this ordination, but the 447 meaning of such measures is questionable on logical grounds. Novel kinds of morphospaces 448 (and morphospace representations) need to be devised to accommodate the problem of 449 inapplicable character states. This would be particularly useful for studies at high taxonomic 450 ranks where OTUs tend to reside in only partially overlapping character spaces. 
The central problem of missing data for disparity analysis is that it generates inconsistencies in the pairwise distance matrix D. If one considers the following three taxa with one missing entry for taxon A for the fifth characters:

Taxon B: $(0,0,1,0,0)$

461 Taxon C: $(0,0,1,0,1)$

Comparison of sequences of B or C with A can only be done for four characters. From this partial information, $\mathrm{C}$ is seen as identical to A, B as identical to A, and yet, B and C differ by one mismatch. A taxon with missing data directly affects the $n$ - 1 distances it is involved in. It

466 does not affect other pairwise comparisons, but it leads to inconsistencies in the overall 467 distance matrix with cases as described above and a proportion of triplets of taxa whose 468 pairwise comparisons do not satisfy the triangle inequality. If so, $\mathbf{D}$ is not fully metric anymore and its PCoA generates negative eigenvalues and potentially confusing visualizations (e.g. morphologically distinct taxa merged into a unique position). Some 471 methods have been proposed to eliminate negative eigenvalues. These methods either add a 472 specific constant value to the non-diagonal entries of $\mathbf{D}$ or $\mathbf{D}^{2}$ (e.g. Lingoes 1971; Cailliez 473 1983) or 'squeeze' the eigenvalues of the Gower-centred matrix (e.g., Hayes and Hill 1981; 474 Kirkpatrick and Lofsvold 1992). These corrections however differentially rescale 
dissimilarities depending on their magnitudes (Legendre and Legendre 1998), and therefore need to be considered carefully in the context of disparity.

477 Obtaining a precise description of the effect of missing data on the distance matrix is a 478 difficult task because numerous factors may affect, in interrelated ways, the response of the 479 distance matrix to missing data. These factors include the shape of the frequency distribution 480 of the pairwise distances (e.g., mean and variance), the shape of the character matrix $\mathbf{M}(n / p$ 481 ratio), the distribution of missing data in $\mathbf{M}$ (amount and structure), and the use of scaling 482 option for dissimilarities (when missing data occur, scaling is employed to make comparisons of dissimilarities fairer when these have been calculated from sequences of character states of different lengths. For instance, observed matches and mismatches between two taxa provide a dissimilarity measure which is then divided by the number of characters involved in its calculation - "mean character difference"; Sneath and Sokal 1973; Foote 1992). The following simulation can help get a glimpse of the impact of missing data on the distance matrix. It is again a simplification of most real case studies, but it supplies an intuitive idea of the average behaviour of $\mathbf{D}$ while leaving aside the idiosyncrasies of empirical datasets. Let $\mathbf{D}$ be the Hamming distance matrix of a complete dataset (taken as a uniform distribution of $n$ 491 taxa in a $p$-hypercube), $\mathbf{D}_{k}$ the distance matrix of the same dataset but plagued with a proportion $k$ of uniformly distributed missing entries, and $\mathbf{D}_{\text {Euc }}$ the Euclidean distance matrix calculated from the principal coordinates of $\sqrt{ } \mathbf{D}_{k}$. Results show that the correlations between

$494 \mathbf{D}$ and $\mathbf{D}_{k}$, and between $\sqrt{ } \mathbf{D}$ and $\mathbf{D}_{\text {Euc }}$, both show a linear decrease with slope -1 as $k$ increases 495 (correlations measured as Pearson's $r$ and Spearman's $\rho$ ). It means for instance that when $k \approx$ $49610 \%, r$ and $\rho \approx 0.9$, and when $k \approx 50 \%, r$ and $\rho \approx 0.5$. Note that in this example, despite the 497 changes in the ranking of distances in $\mathbf{D}_{k}$, morphological disparity measured as the mean 498 pairwise distance (e.g. Foote 1992; Ciampaglio et al. 2001) would on average be left unchanged. This does not occur for meaningful reasons however, but results from the uniform 
distribution of missing data. This random removal of entries in $\mathbf{M}$ has equal probabilities of

501 increasing or decreasing a pairwise distance compared to its true value. As $k$ increases, it also makes greater departures from the true value easier, leading to an increase in the variance of

503 the distribution of distances (with the same mean) which explains the decrease in $r$ and $504 \rho$ observed.

Patterns of missing data

Because of the non-random decay and fossilization of organismal characters (e.g. Sansom et al. 2010), missing data tend not to be randomly distributed in the character matrices but instead preferentially affect suites of characters that are generally lost together for anatomical, structural and/or compositional reasons. Smith et al. (2014) developed a linkage algorithm to account for the resulting differential quality of character scoring and showed that disparity estimates do indeed respond differently to random (Poisson distributed in their simulations)

515 and linked missing data. In addition, taphonomic processes and preservational opportunities can also vary in time and space introducing additional heterogeneities in the pattern of missing entries among taxa.

518 All these factors can artificially inflate or reduce a measure of disparity depending on their 519 particular combination and magnitude for the set of taxa considered. This potentially 520 generates a collection of disparity measures (for instance corresponding to distinct time 521 intervals, geographic areas or taxonomic groups) with reduced commensurability due to the 522 limited overlap of their respective character subspaces. While a small amount of missing data 523 does not seem to prevent reliable measures of disparity, it is unlikely to be the case when the 524 proportion of missing data is significantly higher and heterogeneously distributed. 
525 Unfortunately, the use of phylogenetic data matrices as disparity datasets has been

526 accompanied by a substantial increase in the amount of missing data in disparity studies.

527 Ciampaglio et al. (2001) provided a comparative survey of several disparity indices and

528 investigated their sensitivity to various factors including missing data. At the time, they

529 reported that disparity datasets were typically less than $25 \%$ incomplete and showed that the

530 most widely employed disparity indices were relatively stable up to $30 \%$ of missing data.

531 With the incorporation of phylogenetic datasets within the pool of disparity datasets, this

532 value of $25 \%$ is now regarded as an unrealistic upper bound (Butler et al. 2012; Lloyd 2016).

533 It is not rare to have phylogenetic datasets with more than $50 \%$ of missing data, with frequent

534 instances of taxa with more than 80 or $90 \%$ of their entries unscored (e.g. Butler et al. 2012).

535 Several approaches have therefore been recently suggested to cope with this intensified 536 problem of missing data.

Suggested approaches

541 Phylogenetic correction methods to fill in missing data have been proposed and employed in 542 empirical case studies (Brusatte et al. 2011; Butler et al. 2012). One problem with such 543 approaches is that they are dependent on the phylogenetic hypotheses and impose a model of 544 character evolution on the reconstructed dataset. This might obscure the underlying dynamics 545 of the true disparity signal and impede the test of macroevolutionary hypotheses.

546 Smith et al. (2014) recommended that comparisons of disparity estimates among time 547 intervals that involve sets of taxa differentially affected by missing data should account for 548 this discrepancy. They suggested that taxa should be subjected to removal of some of their 549 character entries, based on an adequate linkage pattern, so that all time intervals have as much 
missing data as the most incomplete one. While this rarefaction procedure might appear

551 sensible, it is unlikely to improve the quality of disparity curves and may in fact decrease the signal-to-noise ratio. Missing entries are detrimental to the measurement of dissimilarity and increasing their amount is not a desirable option. If there are strong variations in the amount

554 of missing data across time intervals, it is preferable not to artificially deteriorate reliable 555 disparity estimates of well documented time intervals for the sake of comparing them to the 556 low quality (and potentially meaningless) disparity estimates of other time intervals.

\section{Recommendations}

561 What other solutions then? Until new fossil discoveries help filling the gaps in the character matrix, there are options to minimize or at least provide reminders of the negative impact of missing data on the measurement of dissimilarities and the interpretation of morphospace and 564 disparity patterns.

565 One approach to lower the relative amount of missing data is to work on subsets of characters.

566 If a pattern of character linkage applies to all the taxa investigated, then it means that the 567 whole dataset can be partitioned into distinct subsets of characters with variable degrees of 568 completeness. Reasonably complete subsets can then be combined or treated separately and 569 their disparity patterns inferred. Beyond the preservational reasons underlying their definition, 570 these subsets of characters might also reflect developmentally or functionally driven 571 associations of traits into coherent units of evolutionary transformation (evolutionary 572 modules; e.g. Schlosser and Wagner 2004). The disparity signals of these subsets might 573 therefore be more helpful for identifying the determinants of a clade's disparity than its global 
disparity signal, because they are less likely to combine characters with different modes and

575 rates of evolution (Hopkins and Gerber 2017).

576 If one is interested in a global disparity signal, then approaches are needed to ensure that the

577 missing data will not mislead the discussions of the results. Again, one risk comes from an

578 undue reliance on morphospace ordination. Back to the hypercube example, if a taxon has $k$

579 entries unscored in its $p$-length character state sequence, it means that the vertex the taxon

580 truly occupies is one within a set of $2^{k}$ vertices, representing a proportion $2^{k-p}$ of the

581 hypercube. As $k$ increases, this can represent a very large set of possible locations (and a large

582 proportion of the total set of vertices when $k$ gets close to $p$ ). Not all possible options are

583 equally likely of course, but still more than one can be expected to be plausible. An increasing

584 proportion of such taxa with high $k$ and high $k / p$ lower the quality of the representation of $\mathbf{D}_{k}$

585 in the principal coordinate space, introducing more and more pronounced discordances

586 between disparity estimates measured from $\mathbf{D}_{k}$ and from the ordination.

587 In general, despite the uneven quality and completeness of the description of taxa in the

588 character matrix, an equal degree of confidence is implicitly attached to all taxa in the

589 morphospace ordination: All of them contribute equally to the definition of the principal

590 coordinate axes; all of them are depicted in the same way as unique points in the ordination

591 space; all of them are considered equally informative in subsequent analyses carried out from

592 the ordination scores. This uneven scoring quality needs to be documented and taken into

593 account. A simple index $q_{i}=\left(p-k_{i}\right) / p$ ranging from 0 to 1 (incomplete to complete) can provide

594 a measure of the quality of the morphological description of taxon $i$.

595 For visualization purposes, it is possible to use a colour-code based on $\mathbf{q}$ (the column vector

596 storing individual values $q_{i}$ ) in order to convey the degree of reliability one can attribute to a

597 given taxon in the morphospace ordination (although its relative position can still be

598 influenced by other taxa). It could also be possible to normalize $\mathbf{q}$ and use it as a vector of 
weights to calculate a weighted covariance matrix $\Sigma$ of the principal coordinate scores. If there is substantial variation in scoring quality among taxa, a principal component analysis of

$601 \Sigma$ then allows a reorientation of the ordination space improving the representation of spatial

602 relationships among the better-preserved taxa (with the reservations expressed earlier

603 regarding the partial information provided by low-dimensional approximations).

604 For the construction of disparity curves, I do not recommend to carry out analyses from the 605 morphospace ordination ("post-ordination disparity" in Lloyd 2016), but instead to obtain 606 disparity measures directly from the distance matrix $\mathbf{D}_{k}$ in order to minimize the impact of 607 missing data. Each time interval is characterized by a set of taxa that is a subset of the total 608 number of taxa sampled. The distance matrix of the subset is therefore a submatrix of $\mathbf{D}_{k}$.

609 Estimating disparity from this submatrix ensures that the estimate is unrelated to the amount 610 and pattern of missing data of taxa occurring in other time intervals. Conversely, a disparity 611 estimate inferred from the principal coordinate scores of the subset of taxa is influenced by all 612 other taxa because the principal coordinate axes are defined from the whole $\mathbf{D}_{k}$. Hence, the 613 detrimental effect of taxa with large amount of missing data will be spread out over the entire 614 time range studied regardless of their temporal occurrence, and affect all ordination-based 615 disparity estimates. Working with submatrices of $\mathbf{D}_{k}$ restricts the impact of poorly-preserved 616 taxa to their own time interval (the same argument holds if one is working with different 617 taxonomic groups or geographic regions instead).

618 Disparity is conceived as the spacing and spread of taxa in morphospace. These features are 619 traditionally calculated as the sum of variances and sum of ranges for a morphospace spanned 620 by continuous variables. For discrete character spaces, the average pairwise distance is 621 conceptually equivalent to the sum of variances (the latter can be shown to be proportional to 622 the average squared pairwise Euclidean distance) and maximum pairwise distance indices 623 analogous to range-based measures can be derived as well (Hughes et al. 2013). The number 
624 of character state combinations is also an intuitive measure of the amount of morphospace

625 occupied provided some of its weaknesses are accounted for (sensitivity to sample size and

626 dimensionality. Thomas and Reif 1993; Foote 1994; Ciampaglio et al. 2001).

627 Finally, it appears essential for disparity studies to systematically report the overall proportion

628 of missing data and the average percentages of unscored entries per taxon and per character.

629 Maximum, minimum, and average values of $q$ (or of any other equivalent quality index)

630 should also be calculated for each time interval and plotted alongside disparity curves. This

631 will provide a helpful indication to decide the level of confidence one can put in the disparity

632 values reported.

633

634

\section{CONCLUDING REMARKS AND PERSPECTIVES}

636

637

On phylogenetic datasets as disparity datasets

638

639 Discrete character spaces have geometric features that make them quite distinct from other

640 types of morphospaces encountered in evolutionary paleobiology. The discrete coding they

641 allow is particularly relevant for the measurement of morphological variation at high

642 taxonomic level. The co-option of phylogenetic data matrices as bases for morphospace

643 construction has significantly increased the frequency of discrete character spaces in disparity

644 studies. A positive outcome has been the broadening of the taxonomic scope of disparity

645 analyses to include many vertebrate clades whose general morphology is difficult to

646 characterize morphometrically. This rapid increase in the use of phylogenetic data matrices

647 nevertheless calls for a critical assessment of their conceptual and operational value. 
648 While it is often stated in these studies that "well established protocols" of disparity analyses

649 were used, it is important to keep in mind that these protocols were devised for much larger

650 datasets (in terms of number of OTUs). For example, the relative insensitivity to sample size

651 of some disparity indices might need to be reconsidered if the number of taxa sampled in a

652 time interval is very low (a frequent result of the temporal partition of small-size phylogenetic

653 data matrices). Likewise, the often-mentioned ability of PCoA to handle missing data

654 undoubtedly has limits, and obtaining principal coordinate scores does not guarantee their

655 meaningfulness if the input distance matrix is poorly representative of the true pattern of

656 variation. Hence, some aspects of the disparity pipeline need to be adjusted or reconsidered in

657 order to account for the specificities of phylogenetic-based disparity datasets and ensure that

658 their use is not associated with disparity inferences of lower quality.

659

660

661

On the measurement of dissimilarity

662

663 Despite the centrality of this problem (the choice of how to relate morphologies underlies the

664 resulting topology of the morphospace), little has been said above on the possible options for

665 measuring dissimilarity between pairs of OTUs. Only a few points are given below (and all

666 assume the validity of a metric topology) but more work and methodological developments

667 are needed in this area (Gerber 2014, 2017).

668 Lloyd (2016) proposed five criteria defining a good distance measure for disparity analyses:

669 (i) distances should be robust against missing data (this property can be assessed by

670 simulations); (ii) the resulting distance matrix should be as Euclidean as possible (leading to

671 no or few negative eigenvalues when carrying out PCoA); (iii) distances should be definable

672 for all possible pairs of OTUs; (iv) their implied spatial pattern should be summarisable with 
673 as few dimensions as possible; (v) the set of distances should be as close to normally

674 distributed as possible (because of the parametric nature of many statistical tests).

675 While points (i) and (iii) are legitimate, points (ii), (iv), and (v) are not justifiable. The choice

676 of the distance measure should be biologically driven and not dictated by the requirements of

677 ordination methods or of any subsequent analyses of the distance matrix. The notion of

678 disparity emerges from the structure of the distribution of distances. Whether the latter departs

679 from normality or does not lend itself to successful ordination in reduced-space should be a

680 secondary concern (patterns of morphospace occupation can be inferred without

681 visualisation). Defining a biologically meaningful measure of distance is a difficult task

682 though given the anatomical diversity of the characters found in phylogenetic and disparity

683 datasets. Weighting approaches might help account for the heterogeneity of characters and of

684 their developmental bases (which invalidates equal weighting and impairs the value of simple

685 measure such as the Hamming distance; see also Mitteroecker and Huttegger 2009). The

686 diverse developmental depth of characters was considered early on as an important issue

687 calling for the differential weighting of characters when measuring dissimilarity between

688 morphologies (Gould 1991). Equal weighting schemes seem to have been preferentially used

689 so far, but treelike representations of development should in principle allow the definition of

690 plausible hierarchies of characters and weighting schemes (Schank and Wimsatt 1986; Arthur

691 1988). The value of such hierarchical approaches needs to be evaluated.

692

693

694 On the visualization of morphospaces

695

696 It has been shown above why a morphospace must be distinguished from its ordination. In the

697 case of discrete character spaces, the ordination consists in a Euclidean embedding of the 
morphospace and the ordination space is thus equipped with a coordinate system that differs

699 from that of the morphospace. This distinction is important and both qualitative descriptions

700 and quantitative analyses of the morphospace should not conflate the two spaces.

701 Furthermore, in most empirical instances of discrete character spaces, the depiction of the

702 spatial relationships among taxa supplied by the ordination is deceptive and its reliability can

703 be further deteriorated by abundant missing data. Some graphical options have been

704 suggested above to limit misinterpretations of morphospace patterns, such as the depiction of

705 the connection structure of the vertices or the drawing of frequency ellipses of the distribution

706 of vertices. It is also possible that the developmentally informed weighting schemes

707 recommended above will improve the graphical representation of morphospaces by providing

708 distributions of pairwise distances better summarized by standard ordination methods.

709 A morphological hypercube has been taken as an example throughout, but many datasets

710 depart from this simple case. If multistate characters are present, the discrete space will be

711 geometrically more complex than a hypercube and its architecture will depend on the

712 ordered/unordered status of the character states. While this architecture might be more

713 difficult to visualize, the options described above can be extended to these more complex

714 cases (e.g. visualization of neighbouring vertices). Finally, obtaining informative

715 visualizations of multidimensional spaces is a very general problem (e.g. Abramson and

716 Zanette 2003) and methods developed in different research fields might be fruitfully adapted

717 to the context of morphospaces.

On missing data 
Missing data appear to be the most serious problem brought on by the increasing popularity of

723 discrete character data in disparity studies, even though its consequences are often

724 overlooked. Numerous interacting biological and physical factors can be responsible for missing entries in discrete character matrices, making their specific effect on disparity estimates difficult to appreciate. Numerical or, ideally, analytical approaches are needed to better characterize the behaviour of dissimilarity and disparity measures in face of these perturbations. Progresses in this area could also help devise ways to lessen the impact of missing data. Meanwhile, reporting the structure (e.g. row and column distributions) and amount of missing data will allow a critical appraisal of the disparity patterns documented. Combined with the small sample size issue resulting from the temporal partitioning of phylogenetic data matrices, missing data can lead to poorly supported disparity curves and temporal shifts in disparity between successive time intervals might not reflect any biological signal. In such contexts, coarser but more robust descriptions of clade shape seem preferable, for instance through the use of a centre-of-gravity approach (e.g. Foote 1991; Hughes et al. 2013). While not as detailed as standard disparity curves, they will still contribute to our assessment of the relative frequencies of possible disparity patterns, and such assessments remain necessary for detecting potential trends and generalities in morphological macroevolution.

\section{Acknowledgements}

I am grateful to Jennifer Hoyal Cuthill and Gunther J. Eble for discussions on some of the topics covered in this contribution and to Zuzana Gerber and Yoland Savriama for comments on an earlier draft. Constructive comments by an anonymous reviewer and a detailed and 
747 insightful review by Peter J. Wagner helped improve and clarify the manuscript. Most of this

748 work was done while holding a research associate position in the Department of Earth

749 Sciences at the University of Cambridge and was funded by a Templeton World Charity

750 Foundation grant (LBAG/143) awarded to Simon Conway Morris.

REFERENCES

755

ABBOTT, E. A. 1884. Flatland: A Romance of Many Dimensions. Seeley \& Co, London, 100 757 pp.

758

ABRAMSON, G. and ZANETTE, D. H. 2003. Two-dimensional projections of a hypercube. Physical Review E, 67, 057101.

761

ARTHUR, W. 1988. A theory of the evolution of development. John Wiley \& Sons Inc., New 763 York, 94 pp.

764

765

BUTLER, R. J., BRUSATTE, S. L., ANDRES, B. and BENSON, R. B. 2012. How do

766 geological sampling biases affect studies of morphological evolution in deep time? A case study of pterosaur (Reptilia: Archosauria) disparity. Evolution, 66, 147-162.

BRUSATTE, S. L., BENTON, M. J., RUTA, M. and LLOYD, G. T. 2008. Superiority, competition, and opportunism in the evolutionary radiation of dinosaurs. Science, 321, 14851488. 
-, MONTANARI, S., YI, H. Y. and NORELL, M. A. 2011. Phylogenetic corrections for

774 morphological disparity analysis: new methodology and case studies. Paleobiology, 37, 1-22.

775

776 CAILLIEZ, F. 1983. The analytical solution of the additive constant problem. Psychometrika,

777

778

779

780

781

782

783

784

785

786

787

788

789

790

791

792

793

794

795

796

797

48, 305-308.

CHARTIER, M., LÖFSTRAND, S., VON BALTHAZAR, M., GERBER, S., JABBOUR, F., SAUQUET, H. and SCHÖNENBERGER, J. 2017. How (much) do flowers vary? Unbalanced disparity among flower functional modules and a mosaic pattern of morphospace occupation in the order Ericales. Proceedings of the Royal Society of London, Series B: Biological Sciences, 284, 20170066.

CIAMPAGLIO, C. N., KEMP, M. and McSHEA, D. W. 2001. Detecting changes in morphospace occupation patterns in the fossil record: characterization and analysis of measures of disparity. Paleobiology, 27, 695-715.

CISNEROS, J. C. and RUTA, M. 2010. Morphological diversity and biogeography of procolophonids (Amniota: Parareptilia). Journal of Systematic Palaeontology, 8, 607-625.

CUNNINGHAM, C. W., OMLAND, K. E. and OAKLEY, T. H. 1998. Reconstructing ancestral character states: a critical reappraisal. Trends in Ecology \& Evolution, 13, 361-366. 
ERWIN, D. H. 2007. Disparity: morphological pattern and developmental context.

800 Palaeontology, 50, 57-73.

801

$802-$ - 2017. The topology of evolutionary novelty and innovation in macroevolution.

803 Philosophical Transactions of the Royal Society B, 372, 20160422.

804

805 ESTABROOK, G. F., JOHNSON Jr, C. S. and MC MORRIS, F. R. 1975. An idealized

806 concept of the true cladistic character. Mathematical Biosciences, 23, 263-272.

807

808 FOOTE, M. 1991. Morphological and taxonomic diversity in clade's history: the blastoid 809 record and stochastic simulations. Contributions from the Museum of Paleontology - The

810 university of Michigan, 28, 101-140.

811

$812-$ - 1992. Paleozoic record of morphological diversity in blastozoan echinoderms. Proceedings

813 of the National Academy of Sciences, 89, 7325-7329.

814

$815-$ - 1993. Discordance and concordance between morphological and taxonomic diversity.

816 Paleobiology, 19, 185-204.

817

818 -. 1994. Morphology of Ordovician-Devonian crinoids. Contributions from the Museum of

819 Paleontology - The university of Michigan, 29, 1-39.

820

$821-$ - 1996. Perspective: evolutionary patterns in the fossil record. Evolution, 50, 1-11.

822

$823-$ - 1997. The evolution of morphological diversity. Annual Review of Ecology and

824 Systematics, 28, 129-152. 
$826-$ 1999. Morphological diversity in the evolutionary radiation of Paleozoic and Post-

827 Paleozoic crinoids. Paleobiology, 25 (S2), 1-115.

828

829 GERBER, S. 2014. Not all roads can be taken: development induces anisotropic accessibility

830 in morphospace. Evolution \& development, 16, 373-381.

831

$832-$ - 2017. The geometry of morphospaces: lessons from the classic Raup shell coiling model.

833 Biological Reviews, 92, 1142-1155.

834

835 GOLOBOFF, P. A., FARRIS, J. S. and NIXON, K. C. 2008. TNT, a free program for 836 phylogenetic analysis. Cladistics 24, 774-786.

837

838 GOULD, S. J. 1991. The disparity of the Burgess Shale arthropod fauna and the limits of

839 cladistic analysis: why we must strive to quantify morphospace. Paleobiology, 17, 411-423.

840

841 GOWER, J. C. 1966. Some distance properties of latent root and vector methods used in

842 multivariate analysis. Biometrika, 53, 325-338.

843

844 HALLIDAY, T. J. D. and GOSWAMI, A. 2016. Eutherian morphological disparity across the 845 end-Cretaceous mass extinction. Biological Journal of the Linnean Society, 118, 152-168.

847 HARRISON, L. and LARSSON, H. C. E. 2015. Among-character rate variation distributions 848 in phylogenetic analysis of discrete morphological characters. Systematic Biology, 64, 307849324. 
HAYES, J. F. and HILL, G. W. 1981. Modification of estimates of parameters in the construction of genetic selection indices ('bending'). Biometrics, 37, 483-493.

HOPKINS, M. J. and GERBER, S. 2017. Morphological Disparity. 1-12. In NUNO DE LA ROSA, L. and MÜLLER, G. (eds). Evolutionary Developmental Biology: A Reference Guide, Springer, Cham.

- and SMITH, A. B. 2015. Dynamic evolutionary change in post-Paleozoic echinoids and the importance of scale when interpreting changes in rates of evolution. Proceedings of the National Academy of Sciences, 112, 3758-3763.

HETHERINGTON, A. J., SHERRATT, E., RUTA, M., WIKINSON, M., DELINE, B. and DONOGHUE, P. C. 2015. Do cladistic and morphometric data capture common patterns of morphological disparity?. Palaeontology, 58, 393-399.

HUGHES, M., GERBER, S. and WILLS, M. A. 2013. Clades reach highest morphological disparity early in their evolution. Proceedings of the National Academy of Sciences, 110, $13875-13879$.

HUNT, G. and SLATER, G. 2016. Integrating paleontological and phylogenetic approaches to macroevolution. Annual Review of Ecology, Evolution, and Systematics, 47, 189-213.

KEARNEY, M. 2002. Fragmentary taxa, missing data, and ambiguity: mistaken assumptions and conclusions. Systematic Biology, 51,369-381. 
KIRKPATRICK, M. and LOFSVOLD, D. 1992. Measuring selection and constraint in the

877 evolution of growth. Evolution, 46, 954-971.

878

879

KLINGENBERG, C. P. and GIDASZEWSKI, N. A. 2010. Testing and quantifying

880 phylogenetic signals and homoplasy in morphometric data. Systematic Biology, 59, 245-261.

881

882

LEGENDRE, P. and LEGENDRE, L. 1998. Numerical Ecology. Elsevier Science,

883

Amsterdam, 853 pp.

884

885

LEWIS, P. O. 2001. A likelihood approach to estimating phylogeny from discrete

886 morphological character data. Systematic Biology, 50, 913-925.

887

888

LINGOES, J. C. 1971. Some boundary conditions for a monotone analysis of symmetric matrices. Psychometrika, 36, 195-203.

890

891

LLOYD, G. T. 2016. Estimating morphological diversity and tempo with discrete character-

892 taxon matrices: implementation, challenges, progress, and future directions. Biological

893 Journal of the Linnean Society, 118, 131-151.

894

895

MADDISON, W. P. 1993. Missing data versus missing characters in phylogenetic analysis.

896

Systematic Biology, 42, 576-581.

897

898 MAYNARD SMITH, J., BURIAN, R., KAUFFMAN, S., ALBERCH, P., CAMPBELL, J.,

899 GOODWIN, B., LANDE, R., RAUP, D. M. and WOLPERT, L. 1985. Developmental

900 constraints and evolution: a perspective from the Mountain Lake conference on development

901 and evolution. The Quarterly Review of Biology, 60, 265-287. 
903 McGHEE, G. R. 2006. The geometry of evolution: adaptive landscapes and theoretical 904 morphospaces. Cambridge University Press, Cambridge, 212 pp.

905

906

MITTEROECKER, P. and HUTTEGGER, S. M. 2009. The concept of morphospaces in 907 evolutionary and developmental biology: mathematics and metaphors. Biological Theory, 4, $908 \quad 54-67$.

909

910 NEIGE, P. 2003. Spatial patterns of disparity and diversity of the Recent cuttlefishes

911 (Cephalopoda) across the Old World. Journal of Biogeography, 30, 1125-1137.

OYSTON, J. W., HUGHES, M., WAGNER, P. J., GERBER, S. and WILLS, M. A. 2015.

914 What limits the morphological disparity of clades?. Interface focus, 5, 20150042.

915

916

PLATNICK, N. I., GRISWOLD, C. E. and CODDINGTON J. A. 1991. On missing entries in 917 cladistic analysis. Cladistics, 7, 337-343.

918

PRENTICE, K. C., RUTA, M. and BENTON, M. J. 2011. Evolution of morphological disparity in pterosaurs. Journal of Systematic Palaeontology, 9, 337-353.

921

RAUP, D. M. 1966. Geometric analysis of shell coiling: general problems. Journal of Paleontology, 40, 1178-1190.

924 paleobiology, Freeman Cooper \& Co, San Francisco, 250 pp. 
RICKLEFS, R. E. 2004. Cladogenesis and morphological diversification in passerine birds.

Nature, 430, 338.

930

ROMANO, M. 2017. Disparity vs. diversity in Stegosauria (Dinosauria, Ornithischia): cranial and post-cranial sub-dataset provide different signals. Historical Biology, 1-9.

RUTA, M., BOTHA-BRINK, J., MITCHELL, S. A. and BENTON, M. J. 2013. The radiation of cynodonts and the ground plan of mammalian morphological diversity. Proceedings of the Royal Society of London, Series B: Biological Sciences, 280, e20131865.

- and WILLS, M. A. 2016. Comparable disparity in the appendicular skeleton across the fishtetrapod transition, and the morphological gap between fish and tetrapod postcrania. Palaeontology, 59, 249-267.

SANSOM, R. S., GABBOTT, S. E. and PURNELL, M. A. 2010. Non-random decay of chordate characters causes bias in fossil interpretation. Nature, 463, 797.

SCHANK, J. C and WIMSATT, W. C. 1986. Generative Entrenchment and Evolution. 3360. In FINE, A. and MACHAMER, P. K. (eds). Proceedings of the Biennial Meeting of the Philosophy of Science Association 1986, East Lansing, Michigan. 384 pp.

SCHLOSSER, G. and WAGNER, G. P. (eds.). 2004. Modularity in Development and

950 Evolution. University of Chicago Press, Chicago, 600 pp. 
955 SMITH, A. J., ROSARIO, M. V., EITING, T. P. and DUMONT, E. R. 2014. Joined at the 956 hip: Linked characters and the problem of missing data in studies of disparity. Evolution, 68 , $957 \quad 2386-2400$.

958

959 SNEATH, P. H. A. and SOKAL, R. R. 1973. Numerical Taxonomy, W.H. Freeman \& co, San $960 \quad$ Francisco, 588 pp.

961

962 STAYTON, C. T. 2015. The definition, recognition, and interpretation of convergent 963 evolution, and two new measures for quantifying and assessing the significance of 964 convergence. Evolution, 69, 2140-2153.

965

966 STRELIN, M. M., BENITEZ-VIEYRA, S., FORNONI, J., KLINGENBERG, C. P. and 967 COCUCCI, A. 2018. The evolution of floral ontogenetic allometry in the Andean genus 968 Caiophora (Loasaceae, subfam. Loasoideae). Evolution \& development, 20, 29-39. 969

970 SWOFFORD, D. L. 2003. PAUP*. Phylogenetic analysis using parsimony (*and other 971 methods), Version 4. Sinauer Associates, Sunderland, MA.

972

973 THOMAS, R. D. K. and REIF, W. E. 1993. The skeleton space: a finite set of organic 974 designs. Evolution, 47, 341-360.

975

976 THORNE, P. M., RUTA, M. and BENTON, M. J. 2011. Resetting the evolution of marine 977 reptiles at the Triassic-Jurassic boundary. Proceedings of the National Academy of Sciences, 978 108, 8339-8344. 
980 WAGNER, G. P. and LYNCH, V. 2010. Evolutionary novelties. Current Biology, 20, R48$981 \quad$ R52.

982

983

WAGNER, P. J. 1997. Patterns of morphologic diversification among the Rostroconchia.

984

Paleobiology, 23, 115-150.

985

986 -. 2010. Paleontological perspectives on morphological evolution. 451-479. In BELL, M. A., 987 FUTUYMA, D. J., EANES, W. F. and LEVINTON, J. S. (eds). Evolution Since Darwin: The 988 First 150 Years. Sinauer Associates, Sunderland, MA, 688 pp.

989

$990-$ - 2012. Modelling rate distributions using character compatibility: implications for 991 morphological evolution among fossil invertebrates. Biology Letters, 8,143-146.

992

993

WAGNER, P. J., RUTA, M. and COATES, M. I. 2006. Evolutionary patterns in early 994

tetrapods. II. Differing constraints on available character space among clades. Proceedings of 995 the Royal Society of London, Series B: Biological Sciences, 273, 2113-2118.

996

997

WILKINSON, M. 1995. Coping with abundant missing entries in phylogenetic inference

998 using parsimony. Systematic Biology, 44, 501-514.

999

WILLS, M. A. 2001. Morphological disparity: a primer. 55-144. In ADRAIN, J. M., EDGECOMBE, G. D. and LIEBERMAN B. S. (eds). Fossils, phylogeny, and form: an analytical approach. Springer, Boston, $402 \mathrm{pp}$.

1003 
FIG. 1. Morphospace and ordination in reduced-space. A, the traditional depiction of

1010 morphospace, here by means of principal coordinate analysis. Taxa are represented as points

1011 in a space of continuous quantitative variables (1: Batrachotomus, 2: Effigia, 3: Prestosuchus, hypercube, is shown by drawing the edges connecting neighbouring vertices. $\mathrm{C}$, another visualization of the morphospace where only a subset of edges is represented. The subset is defined to ensure the complete connectedness of the occupied vertices. The size of points is proportional to the score on the third principal coordinate axis (orthogonal to the plane

1020 defined by the first two principal coordinate axes). The circular ellipse circumscribes $\sim 95 \%$ of

1021 the locations of possible morphologies. D, mean variation of the density of vertices along a principal coordinate axis under 500 random orientations of the hypercube. The black line is the average of the 500 runs represented in grey.

1026 FIG. 2. The mapping of phylogenetic tree onto morphospace. A, a simple phylogenetic hypothesis relating five taxa $\{\mathrm{A}, \mathrm{B}, \mathrm{C}, \mathrm{D}, \mathrm{E}\}$ and corresponding to the most parsimonious tree associated with the discrete character matrix shown on the right. The latter includes 3 parsimony informative characters and 5 autapomorphic characters. B, the visualization of the 
1030 tree in the ordination space when the estimates of ancestral states at internal nodes $\{\mathrm{f}, \mathrm{g}, \mathrm{h}, \mathrm{i}\}$

1031 properly account for the discrete architecture of the morphospace, a 8-hypercube (inferred

1032 ancestral states are displayed in the matrix of the first panel). $\mathrm{C}$, the visualization of the tree

1033 when the ancestral states are incorrectly inferred from the continuous Euclidean coordinate

1034 system of the ordination space (squared-change parsimony). D, same as B, but the subspace

1035 of parsimony informative characters is highlighted. It is a cube and autapomorphies push the

1036 five taxa away from it and from each other in orthogonal directions. Note that the projection

1037 of the morphospace here does not correspond to a principal coordinate analyses of the matrix

1038 of pairwise distances among $\{\mathrm{A}, \mathrm{B}, \mathrm{C}, \mathrm{D}, \mathrm{E}\}$. It has instead been defined to better visualise

1039 the discrepancy between the two modes of ancestral states reconstruction, the architecture of

1040 the parsimony informative subspace and the effect of autapomorphic characters. Such an

1041 orientation could happen for instance with the presence of other groups of taxa and shows

1042 why reconstructed ancestors can appear "outside" the convex hull of the set of taxa from

1043 which ancestors are inferred.

1044

1045

1046

1047

1048

1049

1050

1051

1052

1053

1054 
1056
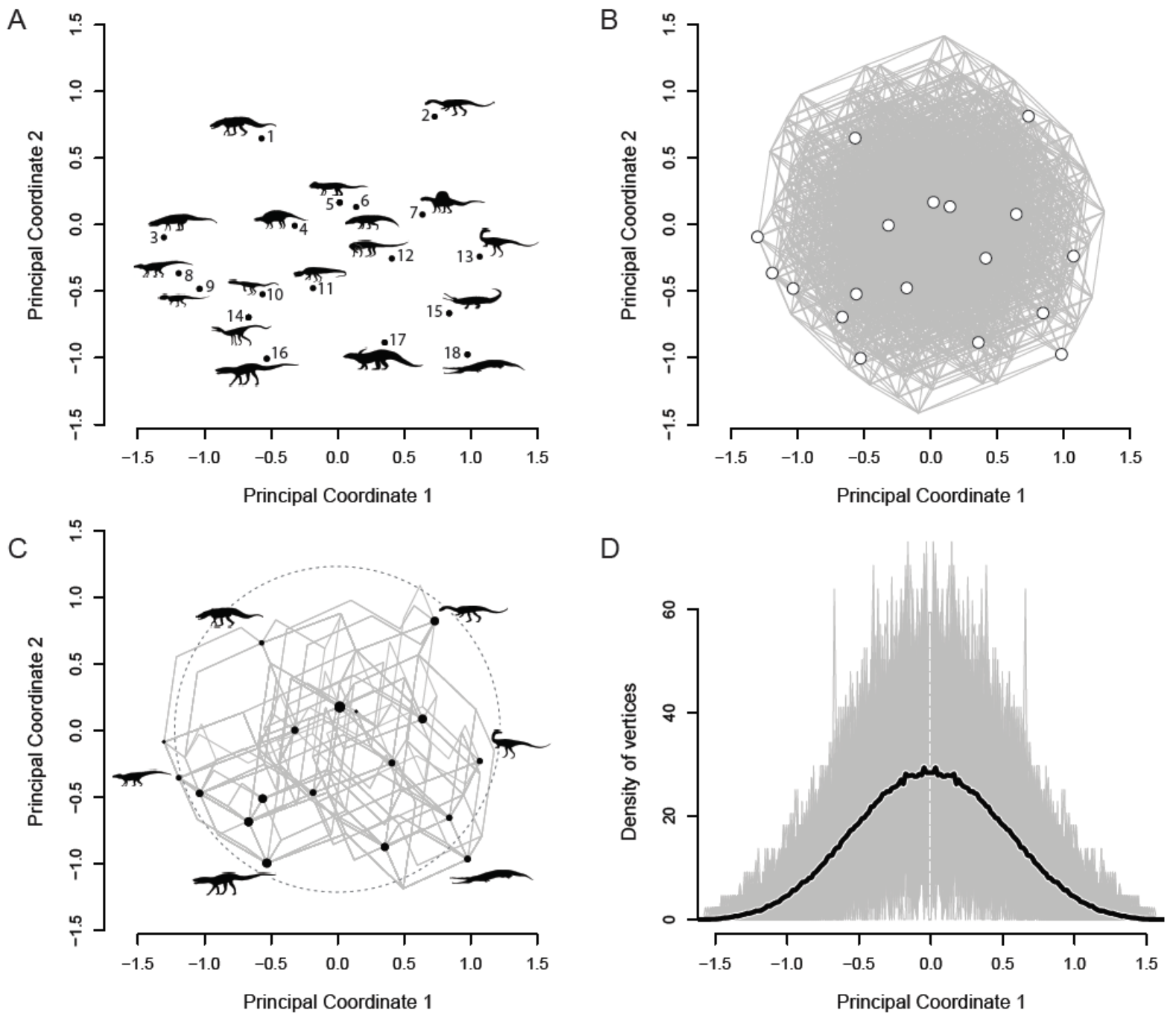
1068

A

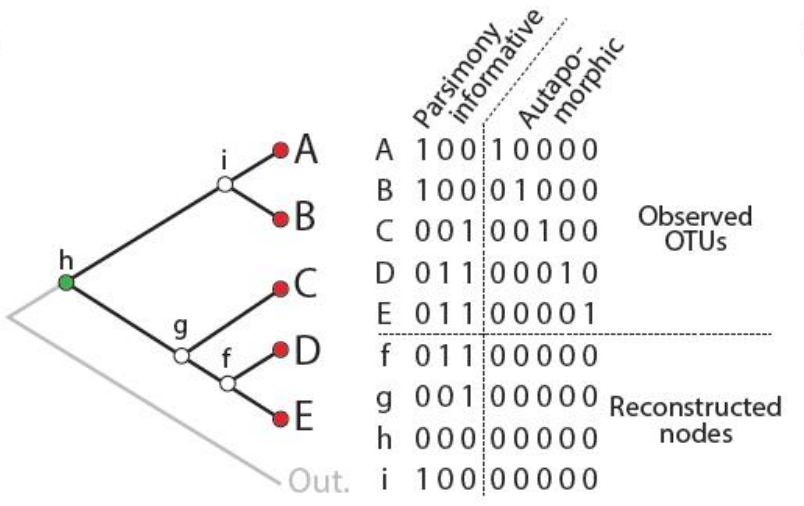

C

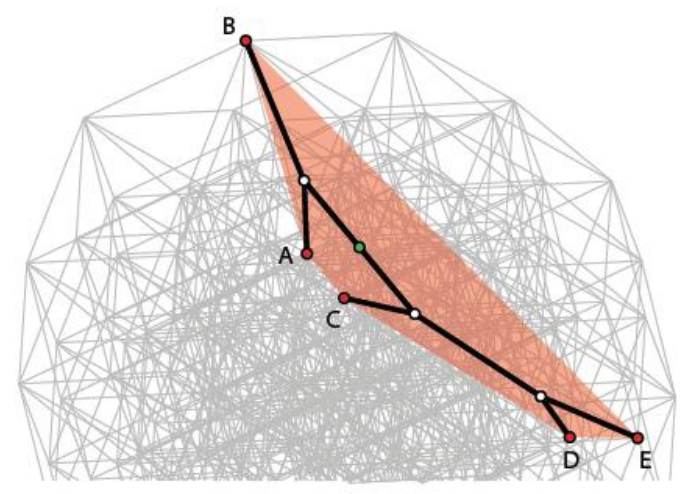

B

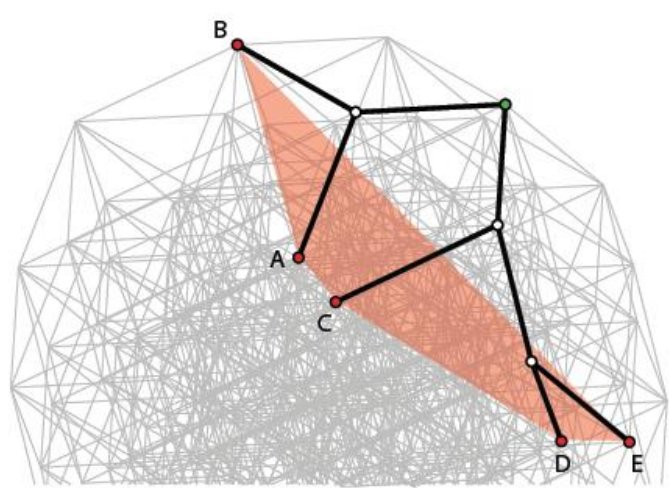

D

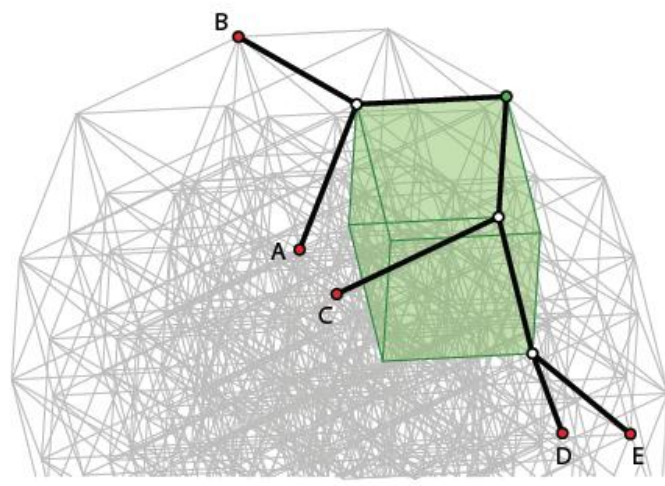

